\title{
Naming Games in Two-Dimensional and Small-World-Connected Random Geometric Networks
}

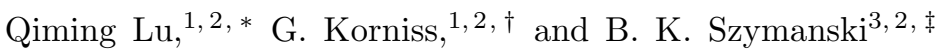 \\ ${ }^{1}$ Department of Physics, Applied Physics, and Astronomy, \\ Rensselaer Polytechnic Institute, 110 8th Street, Troy, NY 12180-3590, USA \\ ${ }^{2}$ Center for Pervasive Computing and Networking, \\ Rensselaer Polytechnic Institute, 110 8th Street, Troy, NY 12180-3590, USA \\ ${ }^{3}$ Department of Computer Science, Rensselaer Polytechnic Institute, 110 8th Street, Troy, NY 12180-3590, USA
}

(Dated: November 21, 2007)

\begin{abstract}
We investigate a prototypical agent-based model, the Naming Game, on two-dimensional random geometric networks. The Naming Game [A. Baronchelli et al., J. Stat. Mech.: Theory Exp. (2006) P06014.] is a minimal model, employing local communications that captures the emergence of shared communication schemes (languages) in a population of autonomous semiotic agents. Implementing the Naming Games with local broadcasts on random geometric graphs, serves as a model for agreement dynamics in large-scale, autonomously operating wireless sensor networks. Further, it captures essential features of the scaling properties of the agreement process for spatiallyembedded autonomous agents. Among the relevant observables capturing the temporal properties of the agreement process, we investigate the cluster-size distribution and the distribution of the agreement times, both exhibiting dynamic scaling. We also present results for the case when a small density of long-range communication links are added on top of the random geometric graph, resulting in a "small-world"-like network and yielding a significantly reduced time to reach global agreement. We construct a finite-size scaling analysis for the agreement times in this case.
\end{abstract}

PACS numbers: 89.75.Fb, 05.65.+b

\section{INTRODUCTION}

Reaching agreement without global coordination is of fundamental interest in large-scale autonomous multiagent systems. In the context of social systems, the objective is to understand and predict the emergence of large-scale population-level patterns arising from empirically supported local interaction rules between individuals (e.g., humans). Examples for such phenomena driven by social dynamics include the emergence and the evolution of languages [1 3 ] or opinion formation [4 -12].

The creation of shared classification schemes in a system of artificial and networked autonomous agents can also be relevant from a system-design viewpoint, e.g., for sensor networks [13, 14]. Envision a scenario where mobile or static sensor nodes are deployed in a large spatially-extended region and the environment is unknown, possibly hostile, the tasks are unforeseeable, and the sensor nodes have no prior classification scheme/language to communicate regarding detecting and sensing objects. Since subsequent efficient operation of the sensor network inherently relies on unique object identification, the autonomous development of a common "language" for all nodes is crucial at the exploration stage after network deployment.

To this end, in this paper we consider and slightly

\footnotetext{
*Electronic address: luq2@rpi.edu

${ }^{\dagger}$ Electronic address: korniss@rpi.edu

¥Electronic address: szymab@rpi.edu
}

modify a simple set of rules, referred to as Language or Naming Games (NG), originally proposed in the context of semiotic dynamics [15, 16]. Such problems have become of technological interest to study how artificial agents or robots can invent common classification or tagging schemes from scratch without human intervention [15, 16]. The original model [15, 17 -19] was constructed to account for the emergence of shared vocabularies or conventions in a community of interacting agents. More recently, a simplified version of the NG was proposed and studied on various network topologies by Baronchelli et al. 20 22], and by Dall'Asta et al. 23, 24] The advantage of studying a minimal model is that one can gain a deeper understanding of the spontaneous selforganization process of networked autonomous agents in the context of reaching global agreement, and can extract quantitative scaling properties for systems with a large number of agents.

In the context of artificial agents, there are other possible scenarios when the NG algorithm, in addition to being interesting in its own merit in studying agreement dynamics on various networks, can also be particularly useful from a system-design viewpoint. That can be the case when one does not intend the outcome of the agreement process among many agents to be easily predictable. The actual process of electing a "leader" or coordinator among sensor nodes may actually be such a scenario. The leader must typically be a trusted node, with possible responsibilities ranging from routing coordination to key distribution [26]. Standard leader election (LE) algorithms 27 31 are essentially based on finding global extremum (e.g., maximum) through local commu- 
nications [27 29]. Thus, the elections can be stolen by placing a node in the network with a sufficiently high ID (e.g., the largest number allowed by the number representation scheme of the sensor chips.) Along these lines, a possible application of the NG algorithm is autonomous key creation or selection for encrypted communication in a community of sensor nodes. Instead of having a centralized or hierarchial key management system with domain and area key distributors [26], group of sensor nodes can elect a key distributor or a security key for secure communications between group members.

This work is an expanded version of our preliminary results [32]. In addition to showing more detailed and extended simulation results, we also study and analyze different aspects of the behavior of the model, in particular, the probability distribution of the agreement times and the cluster-size distribution in the NG on random geometrical graphs (RGGs). Further, we construct and present finite-size scaling for the agreement times in Small-World [33] (SW)-connected RGGs. The remainder of the paper is organized as follows. In Sec. II we briefly review recent results on the NG on various regular and complex networks. In Sec. III we define and present results on the NG with local broadcast on RGGs, motivated by communication protocols in sensor networks. In Sec. IV we present and discuss results on the NG on SWconnected RGGs. Section V concludes our paper with a brief summary and outlook.

\section{BACKGROUND AND PRIOR RESULTS ON THE NAMING GAME}

In the simplified version of the NG, agents perform pairwise games in order to reach agreement on the name to assign to a single object. This version of the NG was investigated on fully-connected (FC) (also referred to as mean-field or homogeneous mixing) [20, 21], on regular [22], on small-world (SW) 23, 34], and on scale-free networks [24, 25]. In the FC network, each agent has a chance to meet with all others and compare their current local vocabularies (list of "synonyms") before updating them. On regular networks, agents have only a limited and fixed number of neighbors on a one-, two-, etc., dimensional grid with whom they can interact/communicate. The communication in both cases is "local", in that pairs of agents are selected to interact and to update their vocabularies. The basic algorithmic rules of the NG are as follows [20, 22]. A pair of neighboring nodes (as defined by the underlying communication topology), a "speaker" and a "listener", are chosen at random [35]. The speaker will transmit a word from her list of synonyms to the listener. If the listener has this word, the communication is termed "successful", and both players delete all other words, i.e., collapse their list of synonyms to this one word. If the listener does not have the word transmitted by the speaker, she adds it to her list of synonyms without any deletion.
Among the above rules, the restriction to a single object 20, 21] strongly reduces the complexity of the model, compared to a more general case where the naming process of multiple objects can be performed simultaneously. From a linguistic viewpoint, this rather strong restriction is equivalent to preventing homonymy, and instead, treating all objects independently. This strong assumption can be more realistic for a system of artificial agents, where agents assign random numbers (e.g., chosen from $2^{31}$ integers) as "words" to new objects. In this case, the number of potential words can be far grater than the number of objects, and the probability that two players invent the same word for different objects (hence giving rise to homonymy) is negligible.

It was found that employing the above local rules (pairwise interactions), after some time, the agents vocabularies converge to a unique vocabulary shared among all agents [20 23]. The major differences between the NG on FC graphs and on regular low-dimensional grids arise in the scaling of the the memory needed to develop the common language before convergence occurs, and in the scaling of the time $t_{c}$ needed to reach global agreement. (The memory need in the present context is the typical value of the largest number of words an agent may posses throughout the evolution of the game [20, 22].) In the FC network, the convergence process to global agreement is fast $\left[t_{c} \sim \mathcal{O}\left(N^{1 / 2}\right)\right.$ for $N$ agents], but large memory $\left[\mathcal{O}\left(N^{1 / 2}\right)\right]$ is needed per agent [20]. For a regular twodimensional network (or grid), spontaneous evolution toward a shared dictionary is slow $\left[t_{c} \sim \mathcal{O}(N)\right]$, but the memory requirement per agent is much less severe $[\mathcal{O}(1)]$ 22. When the NG is implemented on Watts-Strogatz 33. SW networks, the agreement dynamics performs optimally in the sense that the memory needed is small, while the convergence process is much faster than on the regular networks $\left[t_{c} \sim \mathcal{O}\left(N^{0.4}\right)\right.$, closer to that of the FC network] 23].

Sensor networks, which are motivating our study, are both spatial and random. As a large number of sensor nodes are deployed, e.g., from vehicles or aircrafts, they are essentially scattered randomly across large spatiallyextended regions. In the corresponding abstract graph, two nodes are connected if they mutually fall within each others transmission range, depending on the emitting power, the attenuation function and the required minimum signal to noise ratio. Random geometric graphs (RGGs), also referred to as spatial Poisson/Boolean graphs, capturing the above scenario, are a common and well established starting point to study the structural properties of sensor network, directly related to coverage, connectivity, and interference. Further, most structural properties of these networks are discussed in the literature in the context of continuum percolation [36 38$]$.

The common design challenge of these networks is to find the optimal connectivity for the nodes: If the connectivity of the nodes is too low, the coverage is poor and sporadic. If the node connectivity is too high, interference effects will dominate and result in degraded 
signal reception 39 43]. From a topological viewpoint, these networks are, hence, designed to "live" somewhere above the percolation threshold. This can be achieved by adjusting the density of sensor nodes and controlling the emitting power of the nodes; various power-control schemes have been studied along these lines [39, 42, 43]. In this paper we consider RGGs in two-dimensions above the percolation threshold, as minimal models for the underlying network communication topology. Further, we consider RGGs with an added small density of "random" long-range links. The resulting structure resembles small-world (SW) networks [33, 44], also well studied in the context of artificial [45, 46] and social systems [44, 47]. The focus of this work is to study the NG algorithm on these spatially-embedded random graphs.

\section{NAMING GAMES ON RANDOM GEOMETRIC NETWORKS}

\section{A. Random Geometric Graphs}

As mentioned above in the Introduction, first we consider random geometric graphs in two dimensions 36 - 38 as the simplest topological structures capturing the essential features of ad hoc sensor networks. $N$ nodes are uniformly random distributed in an $L \times L$ spatial area. For simplicity we consider identical radio range $R$ for all nodes. Two nodes are connected if they fall within each other's range. An important parameter in the resulting random geometric graph is the average degree $\bar{k}$ (defined as the average number of neighbors per node), $\bar{k}=2 \mathrm{~K} / \mathrm{N}$, where $K$ is the total number of links and $N$ is the number of nodes. In random geometrical networks, there is a critical value of the average degree, $\bar{k}_{c}$, above which the largest connected component of the network becomes proportional to the total number of nodes (the emergence of the giant component) [36 38]. For two-dimensional RGGs $\bar{k}_{c} \approx 4.5$ [38]. There is a simple relationship between the average degree $\bar{k}$, the density of nodes $\rho=N / L^{2}$, and the radio range $R$ of the nodes [36 38], $\bar{k}=\rho \pi R^{2}$, which can be used to control the connectivity (average degree) of the network.

\section{B. The Naming Game with Local Broadcast}

We consider the Naming Game on random geometrical graphs. In the original context of the NG, agents try to reach agreement in finding a unique "word" for an object observed by them. In one of the above proposed potential applications, agents try to generate a shared unique key for encrypted communication. For simplicity, we will use the term "word" for the latter as well when describing the algorithm.

Motivated by communication protocols employed by sensor nodes, we modify the communication rules to make them applicable for sensor networks. Instead of pairwise communications, nodes will initiate broadcast (to all neighbors) in a continuous-time asynchronous fashion. In this paper we consider the initial condition when the "vocabulary" of each node is empty. At every elementary time step, a node is chosen randomly out of $N$ nodes (mimicking Poisson asynchrony for large $N$ ). This node (the "speaker") will broadcast a word from her list of "synonyms"; if her list of synonyms is empty, the speaker randomly invents a word; if she already has several synonyms, it randomly chooses one. Her neighbors (the "listeners") compare their vocabularies with the word transmitted by the speaker. If a listener has this word, she considers the communication a success, and she deletes all other words, collapsing her list of synonyms to this one word. If a listener does not have the word transmitted by the speaker, she adds it to her list of synonyms without any deletion. If at least one listener had the word transmitted, the speaker considers it (at least a partial) success, and (somewhat optimistically) collapses her list of synonyms to this one word. At every step, the "success" rate $S$ is defined as the fraction of listeners who were successful (i.e., those that had the word transmitted by the speaker). From the above it is clear that one of the successful listeners, if any, has to report the outcome of the "word matching" to the speaker. In order to achieve that efficiently, in real sensor-network implementations one can employ the "lecture hall" algorithm [48, 49]. In this paper time $t$ is given in units of one "speaker"-initiated broadcast per node. The main difference between the above algorithm and the one in Refs. [20 23] is the broadcast (instead of pairwise communications) and the underlying network (RGG in this paper) to capture the essential features of the NG in sensor networks.

When starting from empty vocabularies, agents invent words randomly. After time of $\mathcal{O}(1)$ [on average one speaker-initiated broadcast per node], $\mathcal{O}(N /(\bar{k}+1))$ different words have been created. Following the earlytime increase of the number of different words $N_{d}(t)$, through local broadcasts, agents slowly reconcile their "differences", and eventually will all share the same word. First, a large number of small spatial clusters sharing the same word develop. By virtue of the slow coalescence of the interfaces separating the clusters, more and more of the small clusters are being eliminated, giving rise to the emergence of larger clusters, eventually leading to one cluster in which all nodes are sharing the same word. As suggested by Baronchelli et al. [22], this late-time process is analogous to coarsening, a well-known phenomenon from the theory of domain and phase ordering in physical and chemical systems 50]. Figure 1 shows snapshots of vocabularies of the nodes at different times. For later times, group of nodes which already share the same word, slowly coarsen, until eventually only one domain prevails. This behavior is also captured by Fig. 2(b), tracing the number of different words as a function of time $N_{d}(t)$, eventually reaching global agreement, $N_{d}=1$. 


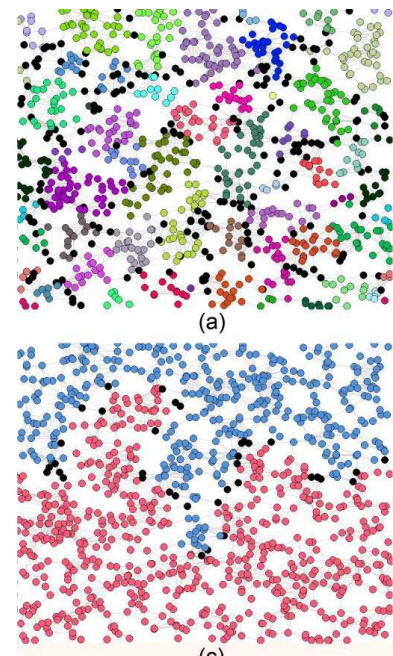

(c)

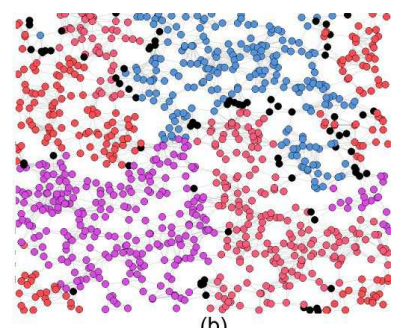

(b)

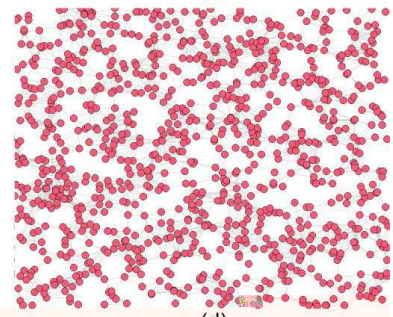

(d)

FIG. 1: (Color) Snapshots of the time evolution of the contents of the agents' word lists during the process of reaching global agreement on RGG for $N=1,000$ nodes at time (a) $t=1$; (b) $t=43$; (c) $t=169$; (d) $t=291$. The average degree is $\bar{k} \approx 12$. Initially, the word lists are empty for all agents. Time, as through the paper, is measured in units of "speaker"-initiated broadcasts per node. Different colors correspond to different words, with black indicating nodes with multiple words. After the early-time increase in the number of different words in the systems, small spatial clusters sharing the same word quickly form, then subsequently "coarsen" until eventually only one global cluster prevails.

\section{Basic Scaling Considerations and Analogy with Coarsening}

Before turning to the detailed discussion of our simulation results, we first sketch the framework of coarsening theory [50], applicable to the observed late-time dynamics of the $\mathrm{NG}$ on regular $d$-dimensional lattices [22]. Coarsening has also been observed in other models relevant to opinion formation and social dynamics [8, 51]. Unlike other minimalist (typically two-state) models often employed to study opinion formation [7], such as the one studied by Sznajd-Weron \& Sznajd 9], the Voter model [51, 52], or the majority rule model [8], in the NG, each agent can be in an unlimited number of discrete states (corresponding to a chosen word). Further, at any instant before reaching global consensus, an agent can have different possible words for the object. Because of the potentially unlimited number of discrete states the agents can assume, the late-stage evolution of the NG resembles that of infinite-state $(Q=\infty)$ Potts model $53-$ 66. .

While RGG is a random structure, it is embedded in two dimensions, and we also attempt to employ elementary scaling arguments from coarsening theory. According to Ref. 22], on regular $d$-dimensional lattices, the typical size of domains (each with already agreed upon one

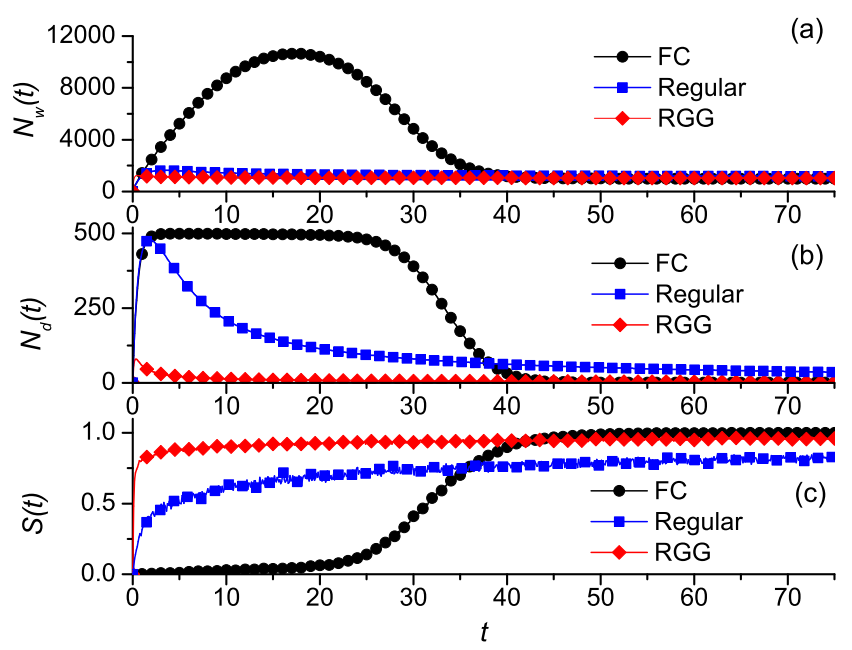

FIG. 2: (Color online) Time evolution of the relevant observables in the Naming Game in the fully-connected (FC), two-dimensional regular (with four nearest neighbors), and random geometric networks (RGG) for $N=1024$, averaged over 1,000 independent realizations; (a) the total number of words in the system $N_{w}(t)$; (b) the number of different words $N_{d}(t)$; (c) the average success rate $S(t)$. The average degree of the underlying RGG is $\bar{k} \approx 12$. Data for the FC and $2 d$ regular networks are reproduced by our simulations, following Refs. 20, 22], for comparison.

word) is governed by a single length scale $\xi(t) \sim t^{\gamma}$ with $\gamma=1 / 2$, analogous to that of domain formation in systems with a non-conserved order parameter 50]. Thus, in $d$ dimensions the average domain size $C(t)$ follows

$$
C(t) \sim \xi^{d}(t) \sim t^{d \gamma}
$$

and the total number of different words $N_{d}$ at time $t$ scales as the typical number of domains

$$
N_{d}(t) \sim \frac{N}{\xi^{d}(t)} \sim \frac{N}{t^{d \gamma}} .
$$

Further, the total number of words $N_{w}\left(N_{w} / N\right.$ being the average memory load per agent), at this late coarsening stage, can be written as the number of nodes $N$ plus the number of nodes with more than one (on average, between one and two) words, separating the different domains. It is of order of typical number of domains times the typical length of the interface of one domain, yielding

$$
N_{w}(t)-N \sim \frac{N}{\xi^{d}(t)} \xi^{d-1}(t) \sim \frac{N}{\xi(t)} \sim \frac{N}{t^{\gamma}} .
$$

Similarly, the "failure rate" for word matching, 1-S(t), (where $S(t)$ is the success rate) scales as the fraction of nodes at the interfaces separating domains with different words

$$
1-S(t) \sim \frac{1}{\xi(t)} \sim \frac{1}{t^{\gamma}}
$$


The main feature of the above power-law decays (up to some system-size dependent cut-offs) is that the number of different words $N_{d}$, the total number of words $N_{w}$, and the success rate $S(t)$ only depend on $t$ through the characteristic length scale $\xi(t)$. Further, for the typical time $t_{c}$ to reach global agreement or consensus, one has $\xi^{d}\left(t_{c}\right) \sim N$, i.e.,

$$
t_{c} \sim N^{1 /(d \gamma)} .
$$

Unless noted otherwise (as in Sec. D.1 and 2), our notation, $t_{c}, N_{d}(t), N_{w}(t), C(t)$, and $S(t)$ refer to the ensemble-averaged values of these relevant observables.

\section{Simulation Results}

Relevant quantities measured in the simulations are the total number of words in the system $N_{w}(t)$ (corresponding to the total memory used by the agents for word allocation at time $t$ ), the number of different words $N_{d}(t)$, and the average size of domains/clusters $C(t)$. Figure 2 displays the time evolution of these three quantities for the RGG, compared to the fully connected (FC) and to the $2 d$ regular networks. Here, for the comparison, we reproduced the corresponding data of Refs. [20, 22]. The behavior of the NG on RGG is qualitatively very similar to that of the NG on $2 d$ regular graphs. After time of $\mathcal{O}(1), \mathcal{O}(N /(\bar{k}+1))$ different words have been invented [Fig. 2(b)]. $\quad N_{w}(t)$ also reaches its maximum in time of $\mathcal{O}(1)$ [Fig. 2(a)]. Focusing on the late-time behavior of the systems, plotting $N_{w}(t) / N-1, N_{d}(t) / N$, and $C(t)$ vs $t$ on log-log scales, confirms the power-law decays associated with the underlying coarsening dynamics, predicted by Eqs. (3), (21), and (10), respectively [Fig. 3]. From the data for $C(t)$, we obtain $2 \gamma=0.79 \pm 0.01$ [Fig. 33(c)], while from the data for $N_{d}(t)$ and $N_{w}(t)$, we extract $2 \gamma=0.74 \pm 0.01$ and $\gamma=0.36 \pm 0.01$, respectively [Figs. 3(b,a)]. Based on our finite-size results, we can only conclude that the coarsening exponent is in the range $0.35<\gamma<0.40$ for the NG on two dimensional RGG. Different exponent values extracted from different observables for finite systems long hindered the precise determination of the coarsening exponent in the closely related large- $Q$ Potts model [56 58]. There, employing advanced Monte Carlo renormalization (MCRG) schemes, it was shown that the coarsening exponent (within error) is $1 / 2$ [58]. However, finite-size effects and very strong transients, in part due to "soft domain walls" and domain-wall intersections ("vertices") can produce values significantly smaller than $1 / 2$ extracted from standard MC methods 56 58], such as ours.

Measuring the time to global agreement, averaged over 1, 000 independent runs (each on a different RGG network realization), we also obtained the scaling behavior of the agreement time, $t_{c} \sim N^{1.10 \pm 0.01}$ and $t_{c} \sim N^{1.07 \pm 0.02}$ for $\bar{k} \approx 12$ and $\bar{k} \approx 50$, respectively, as shown in Fig. 4 . The corresponding scaling exponents both somewhat deviate from the one predicted by Eq. (5) with the exponent

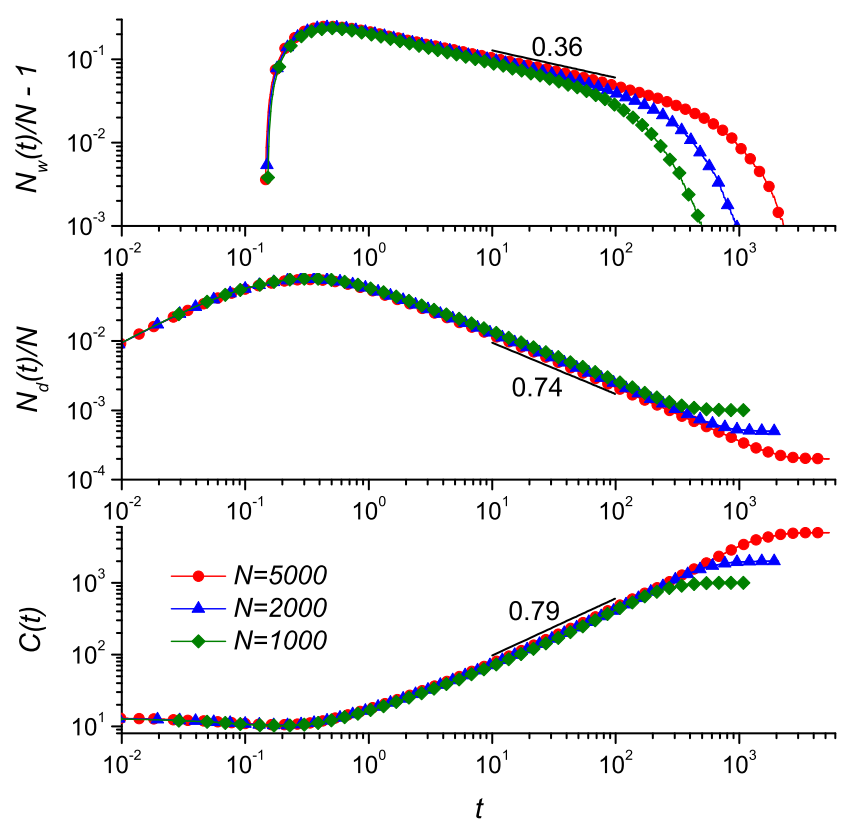

FIG. 3: (Color online) Time evolution of the relevant observables in the Naming Game in random geometric networks (RGG) for three system sizes on log-log scales, averaged over 1, 000 independent realizations; The average degree of the underlying RGGs is $\bar{k} \approx 12$.(a) the normalized total number of words in the system $N_{w}(t) / N-1$; (b) the normalized number of different words $N_{d}(t) / N$; (c) the average domain size $C(t)$. The straight line segments correspond to the best-fit powerlaw decays $N_{w}(t) / N-1 \sim t^{-0.36}, N_{d}(t) / N \sim t^{-0.74}, C(t) \sim t^{0.79}$ for $(\mathrm{a}),(\mathrm{b})$, and $(\mathrm{c})$, respectively.

$1 /(2 \gamma)$. This deviation is possibly due to strong finite-size effects, dominating the very late stage of the agreement dynamics.

For RGGs with many nodes, a relevant control parameter is the average number of neighbors (or average degree) $\bar{k}$. For sensor-network-specific implementations, as noted earlier, $\bar{k}$ can be adjusted by increasing either the density or the communication range of the nodes. We performed simulations of the NG for different average neighborhood size $\bar{k}$, as shown in Fig. The results indicate that the scaling properties (in terms of $N$ ) of the time evolution of the agreement process do not change. The typical convergence times, however, are significantly reduced by increasing the neighborhood size. A closer examination of the convergence time reveals that, for fixed $N$, it scales as $t_{c} \sim \bar{k}^{-2.6}$, in the sparse-network limit $(\bar{k} \ll N)$ in two-dimensional RGGs.

\section{Agreement-time distributions}

In addition to the average agreement (or convergence) time $\left\langle t_{c}\right\rangle$ (time until global agreement is reached), we also

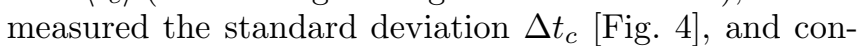
structed the probability density (normalized histograms) 


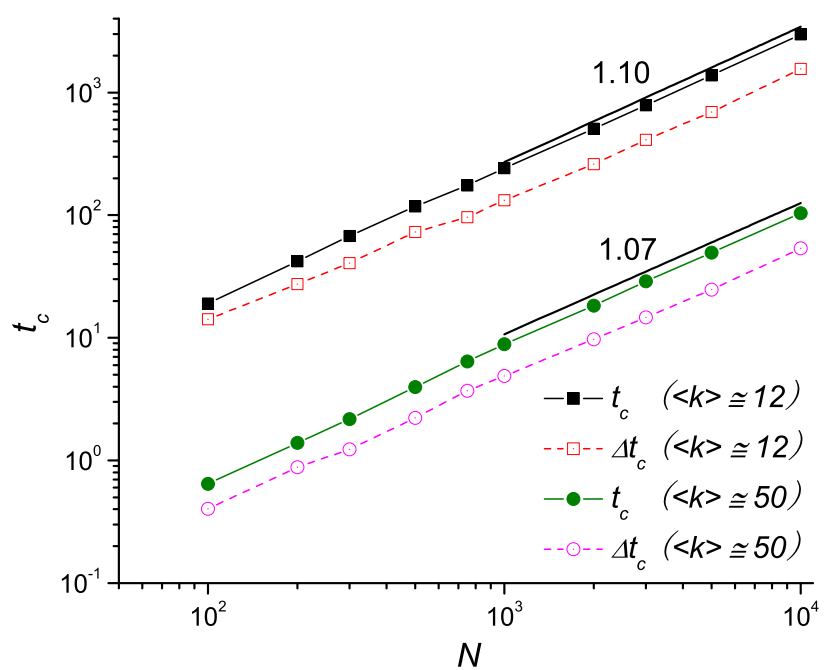

FIG. 4: (Color online) Average and the standard deviation of the convergence time $t_{c}$ until global agreement is reached, as a function of the number of nodes on log-log scales, averaged over 1,000 independent realizations of the NG on RGG. The average degree of the underlying RGGs is $\bar{k} \approx 12$ (squares) and $\bar{k} \approx 50$ (circles). The straight lines correspond to the best-fit power-laws with exponents 1.10, for both the average (solid squares) and the standard deviation (open squares) of RGGs with $\bar{k} \approx 12$, and 1.07 for those of RGGs with $\bar{k} \approx 50$, respectively.

$P\left(t_{c}, N\right)$ for $N$ nodes [Fig. 6]. Since in this subsection, we analyze the full probability density of this observable, we use brackets for denoting the ensemble-averaged value of the convergence time, $\left\langle t_{c}\right\rangle$, while $t_{c}$ alone denotes the stochastic variable, corresponding to a measurement in a single realization of the NG.

Up to the system sizes we could simulate, the standard deviation, within error, scales in the same fashion with the number of nodes as the average itself, $\Delta t_{c} \sim N^{1.10}$ $(\bar{k} \approx 12)$ and $\Delta t_{c} \sim N^{1.07}(\bar{k} \approx 50)$ [Fig. 囵. [Suppressing large average convergence times and the corresponding large standard deviations (through modifying the network communication topology) will be addressed in the next section.]

Further, the shape of the histograms, for sufficiently large systems, remains invariant [Fig. 6]. Thus, introducing the scaled convergence time $x=t_{c} /\left\langle t_{c}(N)\right\rangle$, the corresponding scaled probability densities $p(x)$ for different system sizes collapse onto the same curve. [Fig. [6)(b)]. The above findings indicates that the convergence-time distribution for the NG is governed by a single scale $\left\langle t_{c}\right\rangle$, hence can be written as

$$
P\left(t_{c}, N\right)=\frac{1}{\left\langle t_{c}(N)\right\rangle} p\left(t_{c} /\left\langle t_{c}(N)\right\rangle\right) .
$$

The distributions exhibit exponential tails for large arguments [Fig. [6(b) inset], a characteristic feature of opinion dynamics governed by coarsening $[8,66]$.

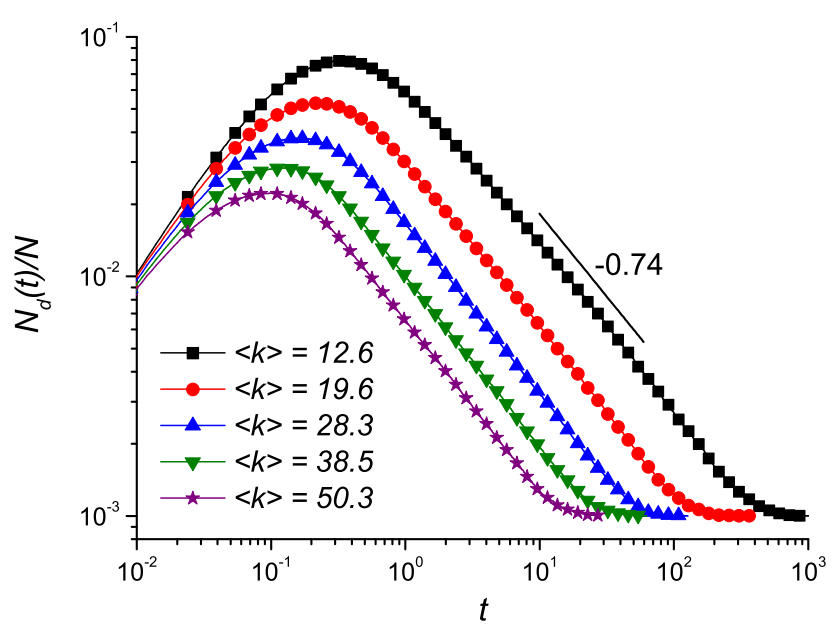

FIG. 5: (Color online) Time evolution of the (scaled) number of different words starting from an "empty" word list initial condition, for various average degree $\bar{k}$ on log-log scales. The number of nodes is $N=1,000$. The straight line segment indicates the asymptotic power-law decay as determined earlier [Figs. 3(b)], independent of the neighborhood size $\bar{k}$.

\section{Cluster-size distributions}

We also studied the probability distribution of the sizes of the clusters during the agreement dynamics $P(C, t)$ (the normalized histogram of the sizes of domains with different words at a given time) [Fig. 7(a)]. Similar to the previous subsection, we analyze the full probability density of this observable, hence we use brackets for denoting the ensemble-averaged value of the cluster size in the system at time $t,\langle C(t)\rangle$, while $C$ alone denotes the stochastic cluster-size variable (sampled at an instant $t$ in a single realization of the NG).

Since the agreement process is governed by coarsening, one expects that this distribution exhibits dynamic scaling, i.e.,

$$
P(C, t)=\frac{1}{\langle C(t)\rangle} p(C /\langle C(t)\rangle)
$$

Thus, $p(x)$, the distribution of the scaled cluster sizes $x=C /\langle C(t)\rangle$ remains invariant for different times. Our simulations confirm this picture, except for very early times (growth phase with initial domains forming) and for very late times (where finite-size effects dominate) [Fig. [7(b)]. The cluster-size distribution exhibit exponential-like tails for large arguments [59 61, 67], as can be seen in Fig. Z(b). 

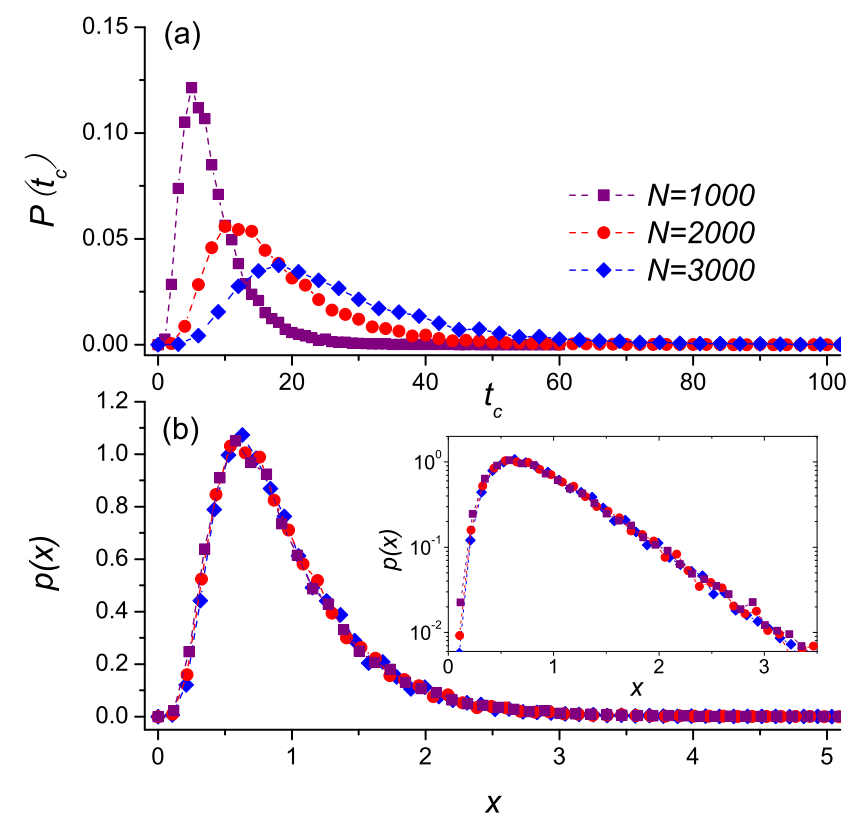

FIG. 6: (Color online) (a) Probability densities of the convergence time for three systems sizes. Data are gathered from 100, 000 independent realizations of the NG on RGG. The average degree of the underlying RGGs is $\bar{k} \approx 50$. (b) Probability densities for the scaled variable $x=t_{c} /\left\langle t_{c}(N)\right\rangle$ for the same data. The inset shows the same scaled histograms on log-lin scales.

\section{NAMING GAMES IN SMALL-WORLD-CONNECTED RANDOM GEOMETRIC NETWORKS}

In light of recent results on NG on one-dimensional SW networks [23], we now consider accelerating the agreement process by adding random long-range communication links between a small fraction of nodes of the RGG. Such networks have long been known to speed up the spread of local information to global scales 33, 44, 47, 68], with applications ranging from synchronization problems in distributed computing [45] to alarm-detection schemes in wireless sensor networks [46]. For sensor networks, this can be implemented either by adding a small fraction of sensors equipped with long-range unidirectional antennas ("physical" long-range connections) or by establishing designated multi-hop transmission patterns ("logical" long-range connections) between certain nodes [69].

We construct the small-world-like RGG (SW RGG) as follows. We start with the original RGG (embedded in $d$ dimensions, where $d=2$ in this paper). Then we add "long-range" links (or "shortcuts") between randomly chosen nodes in such a way that the total number of long-range links per node (the density of random links) is $p$. This $\mathrm{SW}$ construction differs slightly from the original Watts-Strogatz one [33] (also used by Dall'Asta et al. [23]), where random links are introduced by "rewiring"

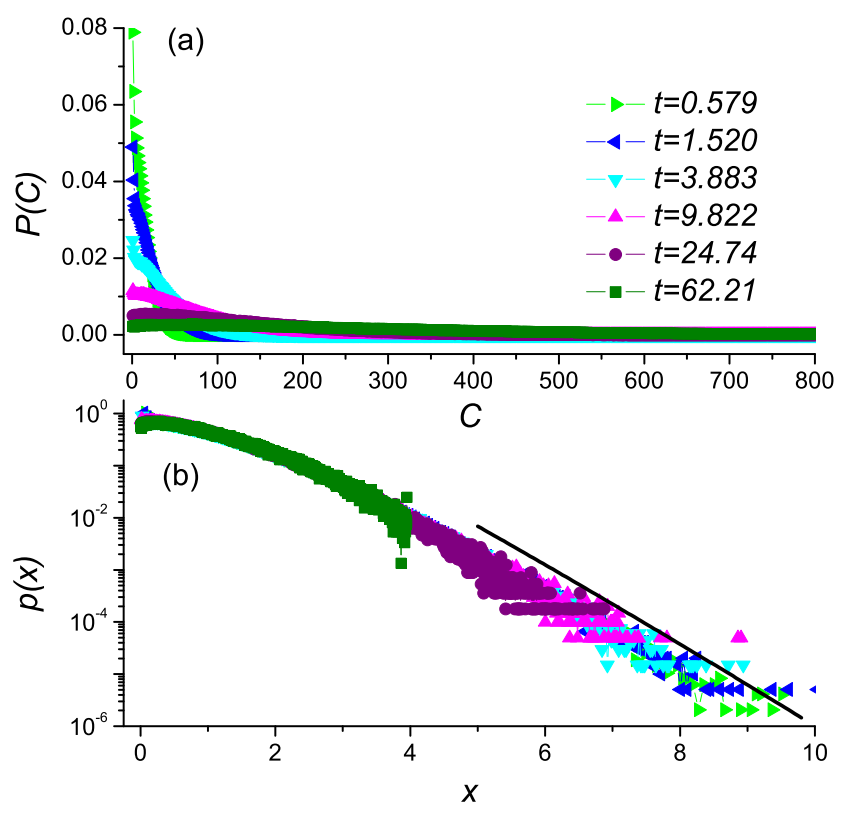

FIG. 7: (Color online) (a) Probability densities of the cluster size at different time of the agreement dynamics. Data are collected through 100, 000 independent realizations of the NG on RGG. The system size of the underlying RGGs is $N=1000$ and the average degree is $\bar{k} \approx 12$. (b) Probability densities for the scaled variable $x=C /\langle C(t)\rangle$ for the same data as in (a) on log-lin scales. The solis curve represents the best fit exponential-like tail, $\propto \exp \left(-1.24 x^{1.12}\right)$.

some of the original connections. The resulting network, however, has the same universal properties in the small$p$, large- $N$ limit [70], which is the center of our interest. Further, it is also motivated by actual implementations in sensor networks, where long-range "channels" are established in addition to the existing local ones.

\section{A. Basic Scaling Considerations}

Before presenting simulation results, using scaling arguments, one can obtain an order of magnitude estimate for the crossover time $t_{\times}$present in the SW RGG and for the time to reach global agreement $t_{c}$ 23]. In SW networks, embedded in $d$ dimensions, the typical (Euclidean) distance between nodes with shortcuts scales as $l_{S W} \sim p^{-1 / d}$ [70 72]. Starting from empty initial word lists word, for early times (following the creation of $\mathcal{O}(N /(\bar{k}+1)))$ different words in the system), the system will exhibit coarsening, until the typical linear size of the growing domains, $\xi(t) \sim t^{\gamma}$, becomes comparable to $l_{S W}$. (Here, both lengthscale measures are understood in terms of the underlying Euclidean metric.) After that time, the agreement process is governed by the presence of random long-range connections, yielding mean-field-like behavior. Hence the crossover from $d$-dimensional coarsening to mean-field-like dynamics occurs when $t^{\gamma} \sim p^{-1 / d}$, yield- 
ing

$$
t_{\times} \sim p^{-1 /(d \gamma)}
$$

In a system of $N$ agents, the above crossover is only displayed if the convergence time of the original system with no random links would exceed the above crossover time $N^{1 / d \gamma} \gg p^{-1 / d \gamma}$, which is equivalent to the condition for the onset of the SW effect $N \gg p^{-1}$ [23, 70]. Following the above system-size independent crossover time, the agreement dynamics is of mean-field like, and one can expect to observe a scaling behavior closer to that of FC networks 20]. In particular, the time to reach global agreement is expected to scale as 23]

$$
t_{c} \sim N^{1 / 2}
$$

a significant reduction compared to that of the "pure" RGG with no long-range links where $t_{c} \sim N^{1.1}$.

\section{B. Simulation Results}

Simulating the NG on SW RGGs qualitatively confirms the above scaling scenario. Following the very early-time development of $\mathcal{O}(N /(\bar{k}+1))$ different words, the system of SW-networked agents, exhibits slow coarsening, with only small corrections to the behavior of the pure RGG [Fig. 8. In fact, this early-time coarsening on SW RGGs is slightly slower compared to pure RGGs due to the effective pinning of interfaces near the shortcuts $4,23,73,74]$. In the NG on SW networks, however, the agreement process only slows down [23], but is not halted by "frozen" (metastable) disordered configurations [4, 73]. After a $p$-dependent crossover time [Eq. (8)], (when the typical size of the growing clusters becomes comparable to the SW length scale), an exponential convergence begins to govern the agreement process. This final-stage fast approach toward consensus sets in earlier for increasing values of the density of shortcuts $p$, yielding a significantly reduced convergence time compared to that of the NG on the "pure" RGG. The temporal behavior of the relevant observables for various values of $p$ can be observed in Fig. 8 .

Plotting the convergence time vs the density of long-range links, as shown in Fig. 9(a), suggests that (for sufficiently large but fixed $N$ ) the convergence time approaches an asymptotic power-law $t_{c} \sim p^{-s}$ with $s=0.79 \pm 0.01$ [23]. On the other hand, for fixed $p$ and increasing $N$, the convergence time increases with $N$, $t_{c} \sim N^{\alpha_{\mathrm{SW}}}$, with $\alpha_{\mathrm{SW}}=0.31 \pm 0.01$ [Fig. 9(b)]. The agreement process is much faster than on a two-dimensional regular grid or RGG and is closer to the anticipated mean-field-like behavior [Eq. (92)] 23]. Thus, in the small-world regime $(N p \gg 1)$ the convergence time depends on both the system size and density of random links, $t_{c} \sim N^{\alpha_{\mathrm{SW}}} / p^{s}$.

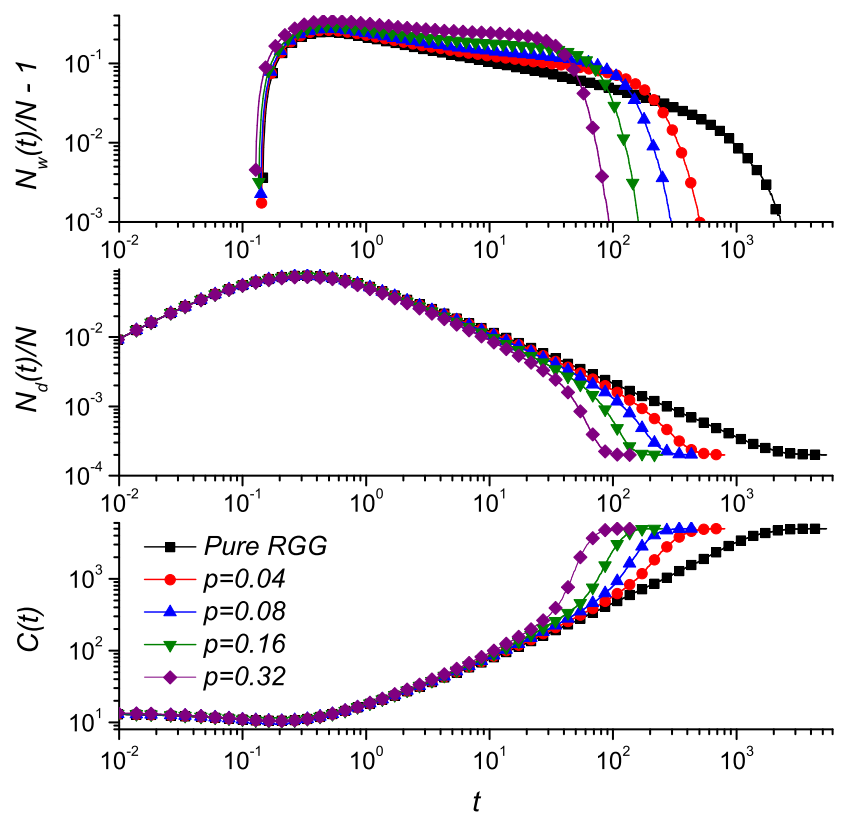

FIG. 8: (Color online) Time evolution of the (scaled) (a) total number of words, (b) number of different words and (c) the average cluster size for SW RGGs on log-log scales, starting from an "empty" word list initial condition, for various density of long-range links $p$, averaged over 1,000 independent realizations of the NG on RGG. The number of nodes is $N=5,000$ with average degree $\bar{k} \approx 12$.

\section{Finite-size scaling for the agreement time on $S W$-RGGs}

In the pure-RGG limit $(N p \ll 1), t_{c}$ only depends on $N$, $t_{c} \sim N^{\alpha_{\mathrm{RGG}}}$ with $\alpha_{\mathrm{RGG}} \approx 1.10$ [Fig. 9(b)] (since, essentially there are no shortcuts in the system). On the other hand, as seen above, in the $\mathrm{SW}$-regime $(N p \gg 1)$, the agreement time scales as $t_{c} \sim N^{\alpha_{\mathrm{SW}}} / p^{s}$. One then can construct the full scaling behavior of $t_{c}(p, N)$, capturing the above two finite-size behaviors as limiting cases on $\mathrm{SW}$-connected RGGs,

$$
t_{c}(p, N) \sim \frac{N^{\alpha_{\mathrm{SW}}}}{p^{s}} f(N p),
$$

where $f(x)$ is a scaling function such that

$$
f(x) \sim\left\{\begin{array}{ll}
x^{s} & \text { if } x \ll 1 \\
\text { const. } & \text { if } x \gg 1
\end{array} .\right.
$$

The pure RGG limit $(N p \ll 1)$ is recovered, provided that $t_{c} \sim\left(N^{\alpha_{\mathrm{SW}}} / p^{s}\right)(N p)^{s} \sim N^{\alpha_{\mathrm{SW}}+s} \sim N^{\alpha_{\mathrm{RGG}}}$, i.e.,

$$
\alpha_{\mathrm{RGG}}=\alpha_{\mathrm{SW}}+s .
$$

Our measured "phenomenological" exponents $\alpha_{\mathrm{RGG}} \approx 1.10, \alpha_{\mathrm{SW}} \approx 0.31$, and $s \approx 0.79$, satisfy the above proposed asymptotic scaling relation. For analyzing our data, Eq. (10) can also be rewritten as

$$
t_{c}(p, N) \sim \frac{(N p)^{\alpha_{\mathrm{SW}}}}{p^{s+\alpha_{\mathrm{SW}}}} f(N p) \sim \frac{1}{p^{\alpha_{\mathrm{RGG}}}} g(N p),
$$



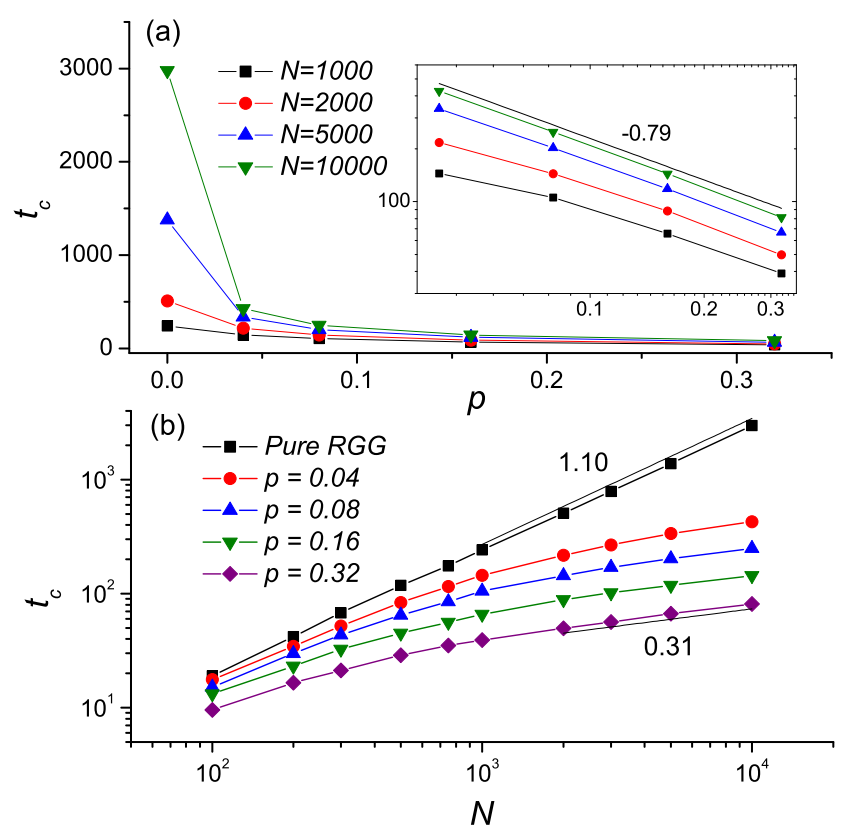

FIG. 9: (Color online) Average convergence time $t_{c}$ for SW RGGs. (a) as a function of the density of shortcuts for various system sizes. The inset shows the same data on log-log scales. The straight lines corresponds to an estimate of the associated (asymptotic) power-law. (b) as a function of the number of nodes on log-log scales for various density of long-range links $p$. The curves shown are obtained by averaging over 1,000 independent realizations of of the $\mathrm{NG}$ on $\mathrm{RGG}$. The average degree of the underlying RGGs is $\bar{k} \approx 12$.

where $g(x)=x^{\alpha_{\mathrm{SW}}} f(x)$. Thus, plotting $t_{c} p^{\alpha_{\mathrm{RGG}}}$ vs $N p$ should yield data collapse, together with the asymptotic small- and large-argument exponents of $g(x), \alpha_{\mathrm{RGG}}$ and $\alpha_{\mathrm{SW}}$, respectively [Fig. 10,

$$
g(x) \sim\left\{\begin{array}{ll}
x^{\alpha_{\mathrm{RGG}}} & \text { if } x \ll 1 \\
x^{\alpha_{\mathrm{SW}}} & \text { if } x \gg 1
\end{array} .\right.
$$

\section{SUMMARY AND OUTLOOK}

In this paper, we studied a prototypical agent-based model, the Naming Game, on Random Geometric Graphs and SW-connected RGGs embedded in two dimensions. While the underlying RGG communication topology is motivated by large-scale sensor networks, the NG on these networks captures fundamental features of agreement dynamics of spatially-embedded networked agentbased systems. We have found that, qualitatively similar to two-dimensional regular networks [22], the NG on RGG can be reasonably well described by the physical theory of coarsening. In particular, local clusters of nodes sharing the same word quickly form, followed by slow coarsening of these clusters in the late stage of the dynamics. The typical length scale grows as $\xi(t) \sim t^{\gamma}$ with

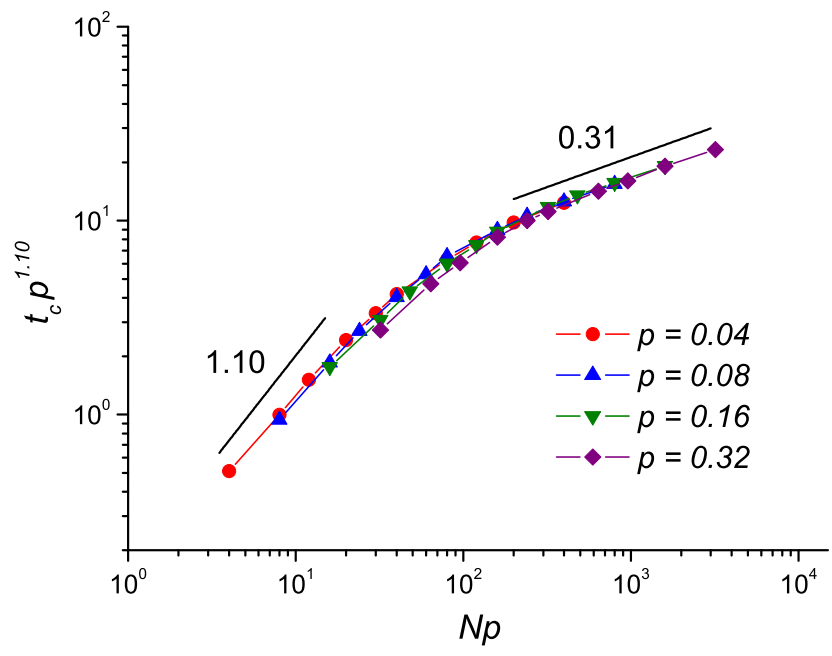

FIG. 10: (Color online) Scaled plot of data shown in Fig. 9 as suggested by the finite-size scaling argument [Eq. (13)]. The straight line segments correspond to the best-fit (asymptotic) power-law behavior of the scaling function $g(x)$ with exponents 1.10 and 0.31 , for small and large arguments, respectively, as described in the text [Eq. (14)].

the coarsening exponent estimated to be $0.35<\gamma<0.40$. Our simulation results also indicate that the average time to reach global agreement is of $\mathcal{O}\left(N^{1.08 \pm 0.03}\right)$ (for fixed average degree). The above results imply that, at least for the range of finite system sizes studied here (up to $N=5,000$ ), the characteristic length scale in twodimensional RGGs grows slower than $1 / 2$. This deviation, in part, may very well be attributed to the effectively small system sizes that we could study. Similarly strong transients and finite-size corrections, due to the presence of "soft domain walls" and "vertices" (domainwall intersections), also made the precise determination of the asymptotic coarsening exponent difficult in the two-dimensional large- $Q$ (effectively $Q=\infty$ ) Potts models [53 58]. On the the other hand, based on our Monte Carlo studies, we cannot rule out the possibility that the deviation from the $\gamma=1 / 2$ coarsening exponent is the result of the inherent local random random structure of RGGs (in contrast to regular two-dimensional grids [22]).

While in this paper we did not address the message complexity of the NG explicitly, one can make an order of magnitude estimate for the typical number of messages needed to reach global agreement on RGGs for an efficient implementation. (In sensor networks, this quantity is also relevant since it corresponds to the global energy consumption.) Once the coarsening process begins, nodes inside the clusters have reached agreement with all their neighbors, of which they are readily aware, hence, they no longer have to initiate broadcasts any longer. Thus, only these "active" nodes, found at the interfaces between these cluster (which have at least one neighbor with different words), will initiate broadcast for 
word matching. Using that the number of nodes at the interfaces scales as $N / t^{\gamma}$ [Eq. (3)], and integrating this expression up to $t_{c} \sim N^{1.08 \pm 0.03}$, one finds that the total number of messages needed to be exchanged until global agreement is reached is of $\mathcal{O}\left(N^{1.68 \pm 0.05}\right)$.

In an attempt to accelerate the agreement process by changing the communication topology between agents, we also studied the SW-connected version of the twodimensional RGG. By adding a small density of shortcuts "on top" of the RGG, resulting in a SW-like network, the convergence time is strongly reduced and becomes of $\mathcal{O}\left(N^{0.31}\right)$, similar to the behavior of NG on the WattsStrogatz SW network [23].

In future works we will investigate the NG on more realistic communication topologies, motivated by and relevant to wireless sensor networks, in particular, random spatial networks with heterogeneous range distribution, minimum-node-degree networks [75], and also networks with dynamically changing connectivities.

\section{Acknowledgments}

We thank B. Yener, J.W. Branch, and A. Barrat for comments on this work. This research was supported in part by NSF Grant Nos. DMR-0426488 (G.K. and Q.L.), NGS-0103708 (B.K.S. and Q.L.), and by Rensselaer's Seed Program. B.K.S. was also supported through participation in the International Technology Alliance sponsored by the U.S. Army Research Laboratory and the U.K. Ministry of Defence under Agreement Number W911NF-06-3-0001.
[1] M. A. Nowak, J. B. Plotkin, and C. Krakauer, J. Theor. Biol. 200, 147 (1999).

[2] M. A. Nowak and N. L. Komarova, Science 291, 114 (2001).

[3] F. A. Matsen and M. A. Nowak, Proc. Natl. Acad. Sci. USA 101, 18053 (2004).

[4] C. Castellano, V. Loreto, A. Barrat, F. Cecconi, and D. Parisi, Phys. Rev. E 71, 066107 (2005).

[5] E. Ben-Naim, Europhys. Lett. 69, 671 (2005).

[6] G. Deffuant, D. Neau, F. Amblard, and G. Weisbuch, Adv. Compl. Syst. 3, 87 (2000).

[7] S. N. Durlauf, Proc. Natl. Acad. Sci. USA 96, 10582 (1999).

[8] P. L. Krapivsky and S. Redner, Phys. Rev. Lett. 90, 238701 (2003).

[9] K. Sznajd-Weron and J. Sznajd, Int. J. Mod. Phys. C 11, 1157 (2000).

[10] B. Kozma and A. Barrat (2007), arXiv:0707.4416.

[11] I.J. Benczik, S.Z. Benczik, B. Schmitmann, and R.K.P. Zia, arXiv:0709.4042 (2007).

[12] T. Antal, P.L. Krapivsky, and S. Redner, Phys. Rev. E 72, 036121 (2005).

[13] Y. Lee, T. C. Collier, C. E. Taylor, and E. E. Stabler, in Proceedings of the 10th International Symposium on Artificial Life and Robotics (2005).

[14] T. C. Collier and C. E. Taylor, Journal of Parallel and Distributed Computing 64, 866 (2004).

[15] L. Steels, Evolution of Communication 1, 1 (1997).

[16] S. Kirby, Artificial Life 8, 185 (2002).

[17] L. Steels, Autonomous Agents and Multi-Agent Systems 1, 169 (1998).

[18] L. Steels, Artificial Life 2, 319 (1995).

[19] L. Steels and A. McIntyre, Advances in Complex Systems 1, 301 (1999).

[20] A. Baronchelli, M. Felici, E. Caglioti, V. Loreto, and L. Steels, J. Stat. Mech.: Theory Exp. p. P06014 (2006).

[21] A. Baronchelli, L. Dall'Asta, A. Barrat, and V. Loreto (2005), e-print arXiv:physics/0511201

[22] A. Baronchelli, L. Dall'Asta, A. Barrat, and V. Loreto, Phys. Rev. E 73, 015102(R) (2006).

[23] L. Dall'Asta, A. Baronchelli, A. Barrat, and V. Loreto,
Europhys. Lett. 73, 969 (2006).

[24] L. Dall'Asta, A. Baronchelli, A. Barrat, and V. Loreto, Phys. Rev. E 74, 036105 (2006).

[25] A. Baronchelli, V. Loreto, L. Dall'Asta, and A. Barrat, in Proceedings of the 6th International Conference on the Evolution of Language, edited by A. Cangelosi, A.D.M. Smith, and K. Smith (World Scientific, Singapore, 2006) pp. 11-18.

[26] B. DeCleene, L. Dondeti, S. Griffin, T. Hardjono, D. Kiwior, J. Kurose, D. Towsley, S. Vasudevan, and C. Zhang, in Proceedings of Military Communications Conference (MILCOM) (2001), vol. 1, pp. 113-117.

[27] D. Angluin, in Proceedings of the 12th ACM Symposium on Theory of Computing (1980), pp. 82-93.

[28] D. S. Hirschberg and J. B. Sinclair, Communications of the ACM 23, 627 (1980).

[29] G. LeLann, in IFIP Congress Proceedings (1977), pp. $155-160$.

[30] N. Malpani, J. Welch, and N. Vaidya, in Proc. Fourth International Workshop on Discrete Algorithms and Methods for Mobile Computing and Communications (2000), pp. 96-103.

[31] S. Vasudevan, B. DeCleene, N. Immerman, J. Kurose, and D. Towsley, in Proc. of the 3rd DARPA Information Survivability Conference and Exposition (DISCEX-III) (2003), vol. 1, pp. 261-272.

[32] Q. Lu, G. Korniss, and B. K. Szymanski, in Proceedings of the 2006 American Association for Artificial Intelligence Fall Symposium Series, Interaction and Emergent Phenomena in Societies of Agents (AAAI Press, Menlo Park, CA 2006) (2006), pp. 148-155; arXiv:cs/0604075v3.

[33] D. J. Watts and S. H. Strogatz, Nature 393, 440 (1998).

[34] B.-Y. Lin, J. Ren, H.-J. Yang, and B. H. Wang (2006), e-print arXiv:physics/0607001

[35] Note that on strongly heterogeneous (scale-free) graphs the order, whether the listener or the speaker is picked first, strongly impacts the efficiency toward global agreement. Picking the listener first at random will increase the chance for picking a node (as a neighbor) with larger degree for speaker. In turn, hubs will be the most fre- 
quent speakers, giving rise to faster convergence to global agreement at a mildly elevated memory cost [24, 25].

[36] R. Meester and R. Roy, Continuum Percolation (Cambridge University Press, 1996).

[37] M. Penrose, Random Geometric Graphs (Oxford University Press, 2003).

[38] J. Dall and M. Christensen, Phys. Rev. E 66, 016121 (2002).

[39] P. Gupta and P. K. Kumar, IEEE Trans. Inf. Theor. IT46, 388 (2000)

[40] F. Xue and P. R. Kumar, Wireless Networks 10, 169 (2004).

[41] B. Krishnamachar, S. B. Wicker, and R. Béjar, in Proceedings of Symposium on Ad-Hoc Wireless Networks (IEEE Globecom, San Antonio, Texas, November, 2001) (2001), vol. 5, pp. 25-29.

[42] W. Krause, I. Glauche, R. Sollacher, and M. Greiner, Physica A 338, 633 (2004).

[43] J. Branch, G. Chen, and B. K. Szymanski, in Proceedings of the International Conference on Networking (ICN 2005), Lecture Notes in Computer Science, Vol. 3420 (Springer-Verlag, New York, 2005), pp. 438-448.

[44] M. E. J. Newman, SIAM Review 45, 167 (2003).

[45] G. Korniss, M. A. Novotny, H. Guclu, Z. Toroczkai, and P. A. Rikvold, Science 299, 677 (2003).

[46] Q. Lu, G. Korniss, and B. K. Szymanski, in Proceedings of the Third International Conference on Networked Sensing Systems (INSS 2006) (Transducer Research Foundation, San Diego, 2006) (2006), pp. 164171.

[47] D. J. Watts, Am. J. Soc. 105, 493 (1999).

[48] G. Chen, J. Branch, and B. K. Szymanski, in Proceedings of the 19th IEEE International Parallel and Distributed Processing Symposium (IPDPS'05) - Workshop 12 (IEEE Computer Society, Washington, DC, 2005), p. 244.1 .

[49] B.K. Szymanski and G. Chen, The Computer Journal (in press, 2008).

[50] A. Bray, Adv. in Phys. 43, 357 (1994).

[51] E. Ben-Naim, L. Frachebourg, and P. L. Krapivsky, Phys. Rev. E 53, 3078 (1996).

[52] T. M. Liggett, Interacting Particle Systems (SpringerVerlag, New York, 1985).
[53] J. A. Glazier, M. P. Anderson, and G. S. Grest, Phil. Mag. 62, 615 (1990).

[54] S. A. Safran, P. S. Sahni, and G. S. Grest, Phys. Rev. B 28, 2693 (1983).

[55] P. S. Sahni, D. J. Srolovitz, G. S. Grest, M. P. Anderson, and S. A. Safran, Phys. Rev. B 28, 2705 (1983).

[56] K. K. Kaski, J. Nieminen, and J. D. Gunton, Phys. Rev. B 31, 2998 (1985).

[57] S. Kumar, J. D. Gunton, and K. K. Kaski, Phys. Rev. B 35, 8517 (1987).

[58] C. Roland and M. Grant, Phys. Rev. B 41, 4663 (1990).

[59] B. Derrida, C. Goudrèche, and I. Yekutieli, Phys. Rev. A 44, 6241 (1991).

[60] B. Derrida and R. Zeitak, Phys. Rev. E 54, 2513 (1996).

[61] P. L. Krapivsky and E. Ben-Naim, Phys. Rev. E 56, 3788 (1997).

[62] C. Sire and S. N. Majumdar, Phys. Rev. Lett. 74, 4321 (1995).

[63] C. Sire and S. N. Majumdar, Phys. Rev. E 52, 244 (1995).

[64] P. A. Alemany and D. ben Avraham, Phys. Lett. A 206, 18 (1995).

[65] T. Masser and D. ben Avraham, Phys. Lett. A 275, 382 (2000).

[66] M. Wykes, Q. Lu, G. Korniss, B. K. Szymanski, and Z. Rácz (2006), to be published.

[67] K. Tafa, S. Puri, and D. Kumar, Phys. Rev. E 63, 046115 (2001).

[68] B. Kozma, M. B. Hastings, and G. Korniss, Phys. Rev. Lett. 95, 018701 (2005).

[69] A. Helmy, IEEE Comm. Lett. 7, 490 (2003).

[70] M. E. J. Newman and D. J. Watts, Phys. Lett. A 263, 341 (1999).

[71] A.Barrat, arXiv:cond-mat/9903323 (1999).

[72] M. Barthélémy and L.A. Nunes Amaral, Phys. Rev. Lett. 82, 3180 (1999); Erratum, Phys. Rev. Lett. 82, 5180 (1999).

[73] D. Boyer and O. Miramontes, Phys. Rev. E 67, 035102(R) (2003).

[74] C. Castellano, D. Vilone, and A. Vespignani, Europhys. Lett. 63, 153 (2003).

[75] Y. Yu, B. Danila, J.A. Marsh, and K.E. Bassler, Europhys. Lett. 79, 48004 (2007). 